\title{
Experimental determination of critical exponents in Anderson localisation of light
}

\author{
C. M. Aegerter, M. Störzer and G. Maret \\ Fachbereich Physik, University of Konstanz - Universitätstrasse 10 \\ 78457 Konstanz, Germany
}

PACS. 42.25.Dd - Wave propagation in random media.

PACS. 05.60.-k - Transport processes.

PACS. 42.25.Bs - Wave propagation, transmission and absorption.

\begin{abstract}
Anderson localisation predicts a phase transition in transport, where the diffuse spread of particles comes to a halt with the introduction of a critical amount of disorder. This is due to constructive interference on closed multiple scattering loops which leads to a renormalisation of the diffusion coefficient. This can be described by a slowing-down of diffusion, where the diffusion coefficient decreases with time according to a power law with an exponent $a$. In the case of strong localisation, where diffusion completely breaks down, the exponent is given by $a=1$. This is due to the fact that such a dependence of the diffusion coefficient naturally leads to a limited spread of the diffusing particle even at infinite times. In the critical regime approaching the transition, a value of $a=1 / 3$ has been predicted, which corresponds to a rescaling of the diffusion coefficient due to the presence of closed loops. Using time-resolved measurements of photon transport in very turbid media, we have determined these scaling exponents experimentally. We find good agreement with theory and determine the critical value of the disorder parameter $k l^{*}$ to be $4.2(2)$. Furthermore, we study the critical exponent of the divergence of the localisation length at the transition, where we find $\nu=1 / 2$, consistent with the expectation for the exponent of an order parameter.
\end{abstract}

Introduction. - Transport through disordered systems can usually be well described by diffusion. However, already in 1958 Anderson showed that for certain systems and high disorder, diffusion breaks down at a phase transition named after him [1]. While Anderson's theory has been applied in the study of the metal-insulator transition, no clear and unambiguous study verifying its predictions has been possible in such systems. This is because electrons traveling through a disordered lattice interact with each other because of their charge and they can also be bound in deep minima of the potential energy landscape [2]. It has been shown subsequently that the wave nature of the diffusing particle is of paramount importance in order to observe Anderson localisation. Therefore, photons traveling through strongly scattering media are an ideal model system with which to study localisation $[3,4]$. This is because photons are not charged and thus do not interact with each other and cannot be bound in deep minima of a random potential. Unfortunately, localisation of photons has not been observed for a long time [5-8], such that an experimental test of the predictions of scaling theory was not possible. The recent observation of signatures of Anderson localisation 
in time-resolved photon transport [9] now does allow a quantitative investigation of the properties of the localisation transition. There are many predictions of localisation theory, which can be tested, such as the value of the critical exponent, $\nu$, with which the localisation length diverges as well as the rescaling of the diffusion coefficient in the critical and localized regimes.

Here, we study the time-resolved transmission of photons and describe them using a modified diffusion theory, where we employ a temporally varying diffusion coefficient, $D$, to take into account localisation effects. Such a local scaling ansatz can describe the subdiffusive nature of transport in the approach to localisation and beyond. This is because the slowingdown of the mean-square displacement, $\left\langle r^{2}\right\rangle$, after a time $\tau_{l o c}$ where the decrease of $D$ sets in, is simply given by $D(t) t$. Thus a localized state can be described by $D(t) \propto 1 / t$ resulting in a finite spread of the photon cloud restricted to $L_{l o c}=\sqrt{D_{0} \tau_{l o c}}$ at all times. This length scale will correspond to the localisation length below the transition and to a correlation length above the transition. In fact the exponent, $a$, with which $D$ decreases can be translated to the rescaling exponent of $D$ with system size following Berkovits and Kaveh [10,11]. Therefore, a determination of $a$ allows an experimental test of the scaling theory of Abrahams et al. [12] for the rescaling of $D$ in the critical regime close to localisation. In addition, we determine $L_{l o c}$ as described above and the mean free path $l^{*}$ from the width of the coherent backscattering cone $[13,14]$. From a combination of these values we can then study the divergence of $L_{l o c}$ at the critical point. Thus we experimentally determine the critical value, where the transition to localisation sets in corresponding to the Ioffe-Regel criterion [15], as well as the exponent $\nu$ with which $L_{l o c}$ diverges. Finally, we use the values of $l^{*}$ and $L_{l o c}$ in order to describe the thickness dependence of the static transmission without any adjustable parameters following the localisation theory of Anderson [4].

Experimental setup. - The experiments described here were carried out on commercially available $\mathrm{TiO}_{2}$ powders from DuPont and Aldrich (Ti-Pure, R700, R706, R101, R104, R900, and R902) with average grain sizes ranging from $220 \mathrm{~nm}$ to $540 \mathrm{~nm}$ and a polydispersity of the order of $20 \%$. The powders are compacted to filling fractions of $\simeq 40 \%$ in order to minimise $l^{*}$. At the wavelength used in the experiment, $590 \mathrm{~nm}$, the particles have a refractive index exceeding 2.7, such that they result in a very turbid medium with very short mean free paths well below $1 \mu \mathrm{m}$. The values of $l^{*}$ are determined directly from the angular width of the enhancement in backscattering direction, which is given by $\left(k l^{*}\right)^{-1}[16]$. Here $k=2 \pi / \lambda$ is the wave number using the free space wavelength. To this end we use a custom-designed setup consisting of 256 highly sensitive photo-diodes fixed to an arc of $1.2 \mathrm{~m}$ diameter [17] resulting in a resolution of $1^{\circ}$ for angles $|\theta|>10^{\circ}$ and $0.15^{\circ}$ for angles $|\theta|<10^{\circ}$. At very small angles, $|\theta|<3^{\circ}$, we also use a setup consisting of a beam splitter and a charge coupled device camera with a resolution of $0.02^{\circ}[18]$. Correcting for internal reflections due to the index mismatch of the turbid medium with the outside air [19], we obtain values of $k l^{*}$ for our samples ranging from 2.5 to 6.3 [9]. The corresponding effective refractive index is calculated using the energy coherent potential approximation [20,21].

The time-resolved transmission is measured using a single photon counting method $[5,22]$. Here a pulsed dye-laser with a pulse width of $\sim 20 \mathrm{ps}$ and a beam waist below $1 \mathrm{~mm}$ is used to illuminate the sample. Then, for each pulse the flight time of a single photon passing through the sample is measured. A histogram of many such pulses leads to a time-of-flight distribution, which is the also the path length distribution of photon paths inside the sample. Due to the presence of after-pulses and the finite width of the pulse, we have to correct the time-of-flight measurements by a deconvolution of the data in Fourier space with a background measurement performed in the absence of a sample. After such a deconvolution, the data can be compared directly to the analytic theory of diffusion of a delta pulse through a slab of 
length $L$ which is given by [22]

$$
T(t) \propto e^{-t / \tau_{a b s}} \sum_{n}(-1)^{n+1} n^{2} \exp \left[-n^{2} \pi^{2} D t / L^{2}\right]
$$

where $\tau_{a b s}$ is the absorption time. At long times, the first term in the sum dominates, such that an exponential decay of the transmission with time is expected. For a localizing sample, where $D$ is scale dependent, this is no longer true and the time dependence in $D(t)$ leads to a non-exponential decay. The effect of a rescaled $D(t)$ on the time-resolved transmission has been calculated by Berkovits and Kaveh, who obtain [10]

$$
T(t) \propto e^{-t / \tau_{a b s}} \sum_{n}(-1)^{n+1}\left(D(t) / D_{0}\right)^{2} n^{2} \exp \left[-n^{2} \pi^{2} D(t) t / L^{2}\right]
$$

Thus the localisation exponent $a$ describing the decay of $D \propto t^{-a}$ can be obtained from the slope of the transmission at long times. At times shorter than the localisation time $\tau_{\text {loc }}$, the time-of-flight distributions are described by classical diffusion theory given in eq. (1). In order to have a fast crossover between the two regimes as indicated by simulations of a selfattracting random walk [23], we have used a function $D(t)=D_{0} \tau_{l o c}^{a} /\left(\tau_{l o c}^{m}+t^{m}\right)^{a / m}$. In the following, the crossover exponent was set to $m=10$. This value does however not matter as long as it ensures a fast enough crossover between a constant and a time-dependent $D$ (i.e. $m>4$ ). Alternatively, the data might be described by the self-consistent theory of Skipetrov and van Tiggelen [24] who calculate the time-resolved transmission for localising samples. Here, a quantitative comparison has so far not been possible since the theory does not include absorption and is limited to thinner samples than we have studied [25]. Qualitatively however, the theory does also predict a temporally varying diffusion coefficient leading to a non-exponential tail in transmission at long times. This decrease in $D$ sets in at the transition to strong localisation.

Results. - Time-of-flight distributions for two of our samples are shown in fig. 1. As can be seen, the panel on the left for a sample with $k l^{*}=2.5$ shows a non-exponential decay indicative of a renormalised $D$ due to localisation. This can also be seen from the fit of localisation theory with an exponent of $a=1$ in the same figure, which describes the data very well. On the other hand, the data on the right panel, at a value of $k l^{*}=4.3$, cannot be described by a fit with $a=1$; however they also show deviations from classical diffusion, see [9]. However, as the fit in the figure show, they are in very good agreement with an exponent of $a=1 / 3$, which is the prediction for the scaling exponent in the critical regime [10].

Such a value for the local scaling exponent translates to a renormalisation of $D$ with sample thickness as $1 / L$, as predicted by Abrahams et al. [12]. Leaving the exponent $a$ as a free fitting parameter for the data, the transition to localisation can be studied directly from the increase of the exponent from its classical value of zero to its localising value of one. This is shown in fig. 2 as a function of $k l^{*}$, where one clearly sees that at low disorder (high $k l^{*}$ ) the exponent is zero and increases to one at a value of $k l_{c}^{*} \simeq 4$. Close to the critical point, the exponent is in the critical regime and shows a value of $1 / 3$ as predicted by scaling theory [10].

For a more quantitative description of the critical point, we have to determine the localisation length $L_{l o c}$ and its divergence as the critical value of $k l^{*}$ is approached. The localisation length can be determined from the time scale $\tau_{l o c}$ at which the description of the time-of-flight distribution changes from the classical case given by eq. (1) to the localising case given by eq. (2). For an exponent of $a=1$, the mean-square displacement of a particle is bound by $\left\langle r^{2}\right\rangle=D \tau_{l o c}$, which corresponds to the localisation length. Above the localisation transition, 

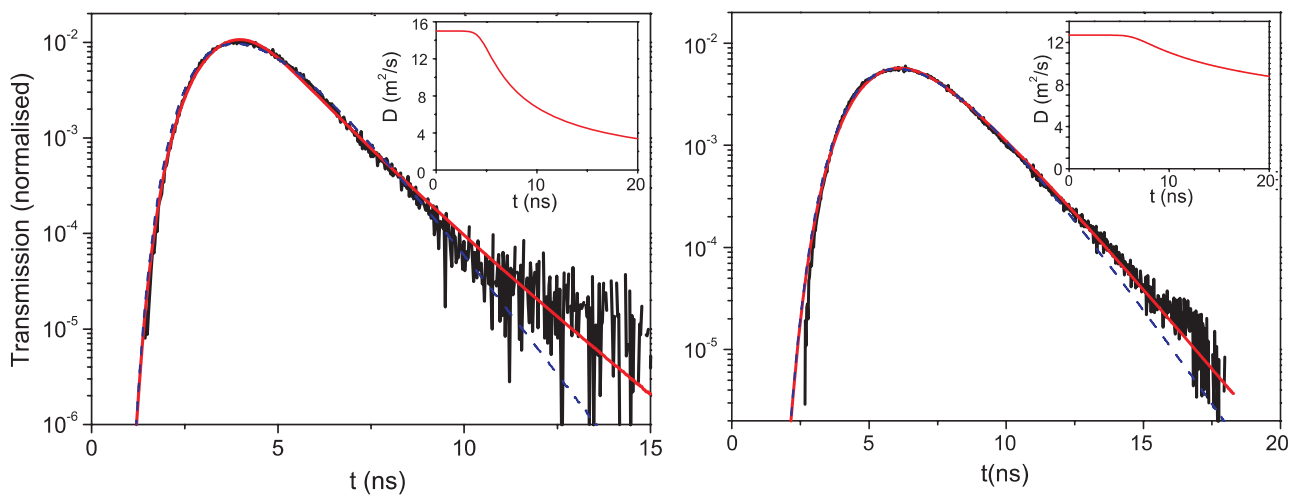

Fig. 1 - Time-of-flight distribution of samples R700 (left; $L=1.21 \mathrm{~mm}, D=15 \mathrm{~m}^{2} / \mathrm{s}, \tau_{a b s}=1.65 \mathrm{~ns}$, $\tau_{l o c}=4.8 \mathrm{~ns}, n=1.55$ ) and R902 (right; $L=1.51 \mathrm{~mm}, D=13 \mathrm{~m}^{2} / \mathrm{s}, \tau_{a b s}=1.44 \mathrm{~ns}, \tau_{l o c}=6.9 \mathrm{~ns}$, $n=1.23$ ). For R700, beyond the localisation transition, the distribution can be well fitted assuming a temporally varying diffusion coefficient proportional to $1 / t$ at times greater than a localisation time, $\tau_{l o c}$ as shown in the inset. This implies that the mean-square displacement of photons comes to a halt at a distance corresponding to $L_{l o c}=\sqrt{D \tau_{l o c}}$. For R902, which is close to the localisation transition, neither classical diffusion nor localisation as used for R700 can fully describe the data. The shown fit assumes a temporal dependence of $D$ shown in the inset as $1 / t^{1 / 3}$, as predicted for the critical regime close to Anderson localisation [10]. This corresponds to a spatial rescaling of $D \propto 1 / L$. For comparison, the fit to classical diffusion [9] is given by the dashed line.

the situation is somewhat more complicated and only a correlation length can be determined due to the finiteness of the sample. Thus for the classical samples, the length scale we determine, $L_{l o c}={\sqrt{D_{0} \tau_{l o c}}}^{a} L^{1-a}$, will be given by the sample thickness $L$. This can be seen in the

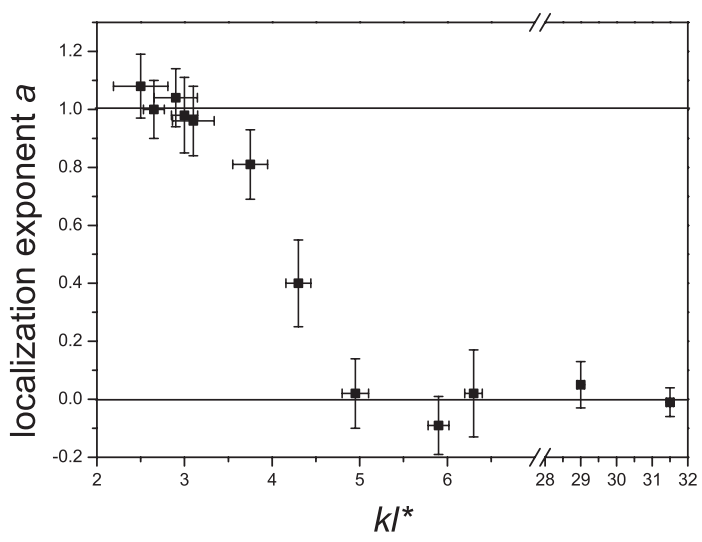

Fig. 2 - The dependence of the localisation exponent as a function of $k l^{*}$. Below the transition to localisation, the exponent is one, such that the spread of photons remains fixed to the localisation length. In the classical regime, the exponent is zero, corresponding to a constant diffusion coefficient. In the critical regime, the diffusion coefficient is renormalised, such that it becomes scale dependent. For a scale dependence $D \propto 1 / L$, the exponent should be $1 / 3$, which is consistent with the data. From the point where the exponent significantly deviates from one, we can also determine the critical value of $k l_{c}^{*}=4.0(2)$. 

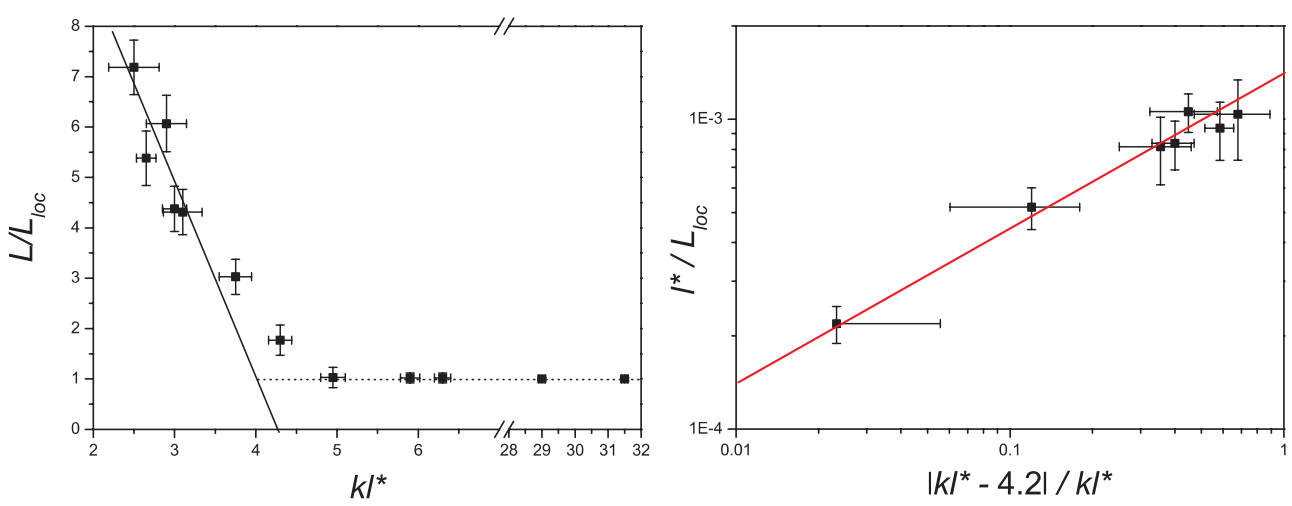

Fig. 3 - The dependence of the inverse localisation length on the critical parameter $k l^{*}$ normalized to the sample thickness is shown on the left. From a linear extrapolation of the data showing a localisation exponent $a \simeq 1$, we obtain a critical value of $k l_{c}^{*}=4.2(2)$. Due to the finite extent of the sample, the determination of the localisation length is limited experimentally to the sample thickness, such that in the classical limit the curve approaches one corresponding to a correlation length above the transition. On the right, the localisation length is normalized to $l^{*}$ and plotted $v s$. the critical parameter $\left|k l^{*}-k l_{c}^{*}\right| / k l^{*}$. This allows the determination of the critical exponent $\nu=0.45(10)$. The straight line in the double logarithmic plot indicates an exponent of $\nu=0.5$.

left panel of fig. 3, where we plot $L / L_{l o c}$ as a function of $k l^{*}$. The figure also shows that a linear extrapolation of the inverse localisation length goes to zero at a value of $k l_{c}^{*}=4.2(2)$, thus determining the critical value for the transition.

Using this estimate of the critical value of $k l^{*}$, we can then determine the critical behaviour of the localisation length at the transition. This is shown in the right panel of fig. 3 , where the inverse of $L_{l o c}$ is normalised to the value of $l^{*}$. The one parameter theory of localisation [12] predicts that this quantity should diverge with a critical exponent $\nu<1$ as

$$
\frac{l^{*}}{L_{l o c}} \propto\left(\frac{\left|k l^{*}-k l_{c}^{*}\right|}{k l^{*}}\right)^{\nu} .
$$

As can be seen from the figure, our data are consistent with a value of $\nu=1 / 2$, with a fitted value of $\nu=0.45(10)$. This exponent corresponds to the inverse slope of the scaling function at the critical point in the one parameter theory [12]. An estimate of the order of magnitude of the slope can be obtained from fig. 1 in [12], which yields a value of $\nu \sim 0.4$ in reasonable agreement with our experimental determination. In fact, an exponent of $\nu=1 / 2$ was predicted on the basis of a mapping of localisation to the dirty $X Y$-model [26]. This is reasonable for the exponent of an order parameter above the critical dimension, which in that case should be four.

With a determination of the absorption and localisation lengths for our samples from timeresolved measurements, it is now also possible to describe the static transmission through a sample using no adjustable parameters. This is shown in fig. 4 for sample R700. For sample thicknesses varying from $0.2 \mathrm{~mm}$ to $2.5 \mathrm{~mm}$, we observe a decrease in transmitted intensity of twelve orders of magnitude that follows an exponential decay. While such a decay is predicted in the case of localisation [4,12], this could also be due to absorption, which also leads to an exponential decay of the transmitted intensity with the decay length given by $L_{a b s}=\sqrt{D \tau_{a b s}}[8]$. However, since we have measured the absorption time in the time-of-flight measurements, as well as the transport mean free path from coherent backscattering, it is 


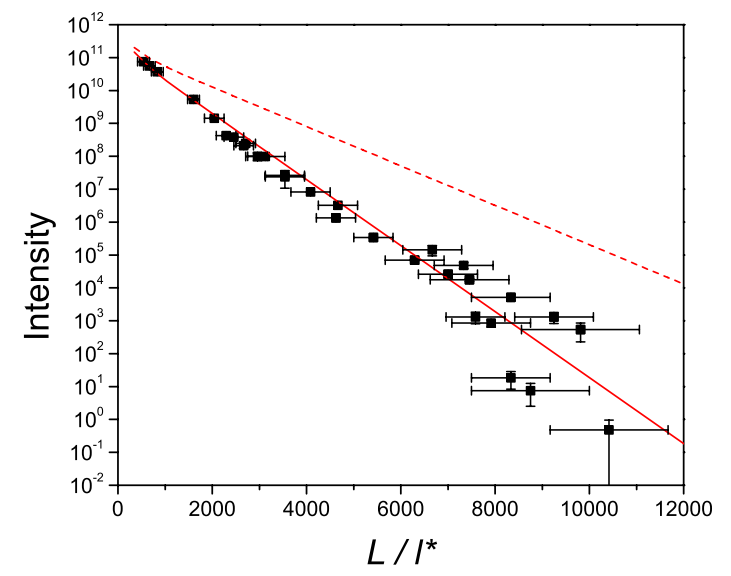

Fig. 4 - The static transmission of the most localizing sample R700 as a function of sample thickness normalised to $l^{*}$. As can be seen, the transmission strongly decreases over twelve orders of magnitude within $2 \mathrm{~mm}$. Using the absorption length obtained from the fit to the time-of-flight distributions in fig. 1 one would expect the dashed line, which decreases much more slowly. Including the localisation length, again determined from the time-of-flight measurements, the full line is obtained, which describes the data without any adjustable parameter. Due to a slight difference in filling fraction of the different samples, there is a corresponding uncertainty in the values of $l^{*}$ indicated by the horizontal error bars.

possible to calculate directly the expectation of the transmitted intensity due to diffusion and absorption [18]. This is shown as the dashed line in the figure and clearly underestimates the decrease in transmission observed experimentally. Adding an exponential decrease with the localisation length from the time-of-flight measurements to the classical expectation, we obtain the full line in fig. 4, which is in good agreement with the data. Note that these calculations were performed without any adjustable parameters and that all the relevant variables were determined experimentally from other measurements. This again shows that the determination of the localisation length from the time-of-flight data is possible and consistent with the predictions of localisation theory [4].

Discussion and conclusions. - The data we have presented show clear evidence of the transition to localisation as described by a rescaled diffusion coefficient [10]. The exponents with which $D$ decreases are in good accord with the predictions of localisation theory and show that the spread of the photon cloud is limited to a finite size at all times. In the critical regime, close to the transition, the diffusion coefficient is renormalised, such that it becomes scale dependent as $D \propto 1 / L$, as was predicted by John and Anderson [3,4]. From the increase of the localisation exponent $a$ to its final value of 1 , we can also obtain an estimate of the critical point at $k l^{*} \simeq 4$, which is in reasonable agreement with the Ioffe-Regel criterion [15]. This can be made more quantitative by the determination of the localisation length $L_{l o c}$ from the time-of-flight data and observing the point of its divergence. The inverse localisation length as shown in fig. 3 decreases towards the critical point at $k l^{*}=4.2(2)$ with an exponent $\nu=0.45(10)$. This is in good accord with the exponent expected for an order parameter, albeit only above the critical dimension [26]. Such an exponent is also in reasonable agreement with the predictions of scaling theory [12], where the inverse slope of the scaling function can be used to estimate $\nu$. While this slope is of the order of 2.5 in fig. 1 of [12], a simple $\epsilon$ expansion would obtain a slope closer to one [3]. Thus our results indicate that a one loop theory is 
probably not enough. These results thus provide a stringent test of localisation theory, where most predictions are found to be fulfilled and any deviations cannot be due to correlation effects [27] or bound states as these do not appear with photons.

This work was supported by the Deutsche Forschungsgemeinschaft, the International Research and Training Group "Soft Condensed Matter of Model Systems" and the Center for Applied Photonics (CAP) at the University of Konstanz. Furthermore, we would like to thank DuPont chemicals and Aldrich for providing the samples used in this study. We would also like to thank P. Gross for support with the cone setup.

\section{REFERENCES}

[1] Anderson P. W., Phys. Rev., 109 (1958) 1492.

[2] Altshuler B. L., Lee P. A. and WebB R. A. (Editors), Mesoscopic Phenomena in Solids (North-Holland, Amsterdam) 1991.

[3] John S., Phys. Rev. Lett., 53 (1984) 2169.

[4] Anderson P. W., Philos. Mag. B, 52 (1985) 505.

[5] Drake J. M. and Genack A. Z., Phys. Rev. Lett., 63 (1989) 259.

[6] van Albada M. P., van Tiggelen B. A., LagendiJK A. and Tip A., Phys. Rev. Lett., 66 (1991) 3132.

[7] Wiersma D. S., Bartolini P., Lagendijk A. and Righini R., Nature, 390 (1997) 671.

[8] Scheffold F., Lenke R., Tweer R. and Maret G., Nature, 398 (1999) 206; see also Wiersma D. S. et al., Nature, 398 (1999) 207.

[9] Störzer M., Gross P., Aegerter C. M. and Maret G., Phys. Rev. Lett., 96 (2006) 063904.

[10] Berkovitz R. and Kaveh M., J. Phys.: Condens Matter, 2 (1990) 307.

[11] Berkovitz R. and Kaveh M., Phys. Rev. B, 36 (1987) 9322.

[12] Abrahams E., Anderson P. W., Licciardello D. C. and Ramakrishnan T. V., Phys. Rev. Lett., 42 (1979) 673.

[13] van Albada M. P. and LagendiJk A., Phys. Rev. Lett., 55 (1985) 2692.

[14] Wolf P. E. and Maret G., Phys. Rev. Lett., 55 (1985) 2696.

[15] Ioffe A. F. and Regel A. R., Prog. Semicond., 4 (1960) 237.

[16] Akkermans E., Wolf P. E. and Maynard R., Phys. Rev. Lett., 56 (1986) 1471.

[17] Gross P., Diploma Thesis (University of Konstanz) unpublished (2005).

[18] Lenke R. and Maret G., in Scattering in Polymeric and Colloidal Systems, edited by Brown W. and Mortensen K. (Gordon and Breach Scientific, New York) 2000, sect. 1.

[19] Zhu J. X., Pine D. J. and Weitz D. A., Phys. Rev. A, 44 (1991) 3948.

[20] Soukoulis C. M. and Datta S., Phys. Rev. B, 49 (1994) 3800.

[21] Busch K. and Soukoulis C. M., Phys. Rev. B, 54 (1996) 893.

[22] Watson G. H., Fleury P. A. and McCall S. L., Phys. Rev. Lett., 58 (1987) 945.

[23] Lenke R., Tweer R. and Maret G., Eur. Phys. J. B, 26 (2002) 235.

[24] Skipetrov S. E. and van Tiggelen B. A., Phys. Rev. Lett., 96 (2006) 043902.

[25] Skipetrov S. E., private communication.

[26] Schuster H. G., Z. Phys., 31 (1978) 99.

[27] Castellani C., Kotliar G. and Lee P. A., Phys. Rev. Lett., 59 (1987) 323. 